\title{
Table des matières
}

Préface

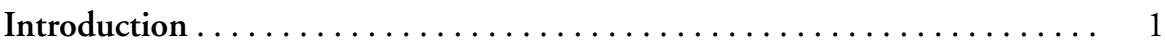

Chapitre 1 Entrer dans la spectroscopie astronomique amateur ..... 7

1.1 Spectrograhie, spectrométrie, spectroscopie... . . . . . . . 7

1.2 À quoi ressemble un spectre? .................. 7

1.3 Le chaînon manquant $\ldots \ldots \ldots \ldots \ldots \ldots \ldots \ldots \ldots \ldots \ldots \ldots \ldots \ldots \ldots \ldots$

1.4 Bref historique $\ldots \ldots \ldots \ldots \ldots \ldots \ldots \ldots \ldots \ldots \ldots \ldots \ldots \ldots \ldots \ldots \ldots \ldots$

1.5 La spectroscopie amateur de nos jours $\ldots \ldots \ldots \ldots \ldots \ldots \ldots$

1.6 Aborder la spectroscopie . . . . . .............. 20

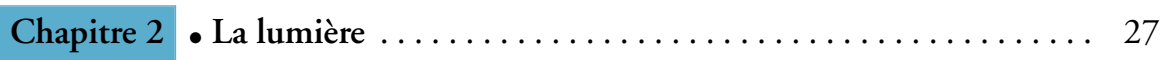

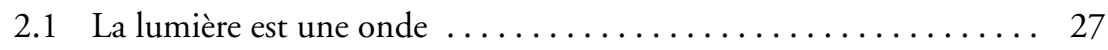


2.2 La lumière est une particule $\ldots \ldots \ldots \ldots \ldots \ldots \ldots \ldots \ldots \ldots \ldots \ldots \ldots$

2.3 Fabriquer de la lumière $\ldots \ldots \ldots \ldots \ldots \ldots \ldots \ldots \ldots \ldots \ldots \ldots \ldots \ldots \ldots$

2.4 Décaler un spectre $\ldots \ldots \ldots \ldots \ldots \ldots \ldots \ldots \ldots \ldots \ldots \ldots \ldots \ldots \ldots \ldots$

2.5 Ce que voit l'œil humain $\ldots \ldots \ldots \ldots \ldots \ldots \ldots \ldots \ldots \ldots \ldots \ldots \ldots \ldots$

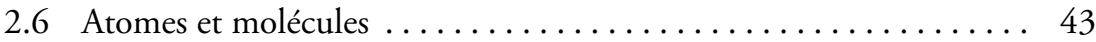

Chapitre 3 • Ce que la lumière nous dit des étoiles . . . . . . . . . 45

3.1 La lumière d'une étoile ordinaire $\ldots \ldots \ldots \ldots \ldots \ldots \ldots \ldots \ldots$

3.2 Chaque étoile a son spectre propre $\ldots \ldots \ldots \ldots \ldots \ldots \ldots \ldots$

3.3 Observer à différentes résolutions . . . . . . . . . . . . . . 49

3.4 Magnitude limite ..................... 53

3.5 Un ciel en mouvement $\ldots \ldots \ldots \ldots \ldots \ldots \ldots \ldots \ldots \ldots \ldots \ldots \ldots \ldots \ldots \ldots \ldots \ldots \ldots$

3.6 Évolution dans le temps $\ldots \ldots \ldots \ldots \ldots \ldots \ldots \ldots \ldots \ldots \ldots \ldots \ldots \ldots \ldots \ldots \ldots$

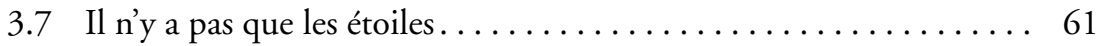

3.8 Une chimie très rudimentaire $\ldots \ldots \ldots \ldots \ldots \ldots \ldots \ldots \ldots \ldots \ldots$

Chapitre 4 • Quelles observations avec quel instrument?......... 63

4.1 Les questions de base $\ldots \ldots \ldots \ldots \ldots \ldots \ldots \ldots \ldots \ldots \ldots \ldots \ldots \ldots \ldots$

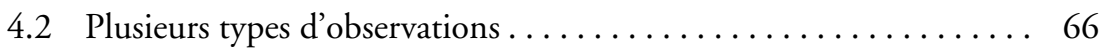

4.3 Quel phénomène physique observer?............. 68

4.4 Commencez par la basse résolution . . . . . . . . . . . 69

4.5 Commencez par les différents types spectraux .......... 70

4.6 Organisez votre observation $\ldots \ldots \ldots \ldots \ldots \ldots \ldots \ldots \ldots \ldots \ldots \ldots \ldots \ldots \ldots \ldots$

Chapitre 5 • Principes optiques d'un spectroscope ........... 71

5.1 Réflexion, réfraction et diffraction $\ldots \ldots \ldots \ldots \ldots \ldots \ldots \ldots \ldots \ldots \ldots$

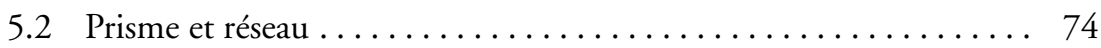

5.3 Rappels d'optique géométrique $\ldots \ldots \ldots \ldots \ldots \ldots \ldots \ldots \ldots . \ldots 4$

5.4 Lunettes et télescopes $\ldots \ldots \ldots \ldots \ldots \ldots \ldots \ldots \ldots \ldots \ldots$

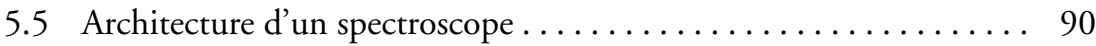

5.6 Un exemple concret : Alpy $600 \ldots \ldots \ldots \ldots \ldots \ldots \ldots \ldots$

5.7 Un autre exemple $:$ Lhires III $\ldots \ldots \ldots \ldots \ldots \ldots \ldots \ldots \ldots \ldots \ldots \ldots \ldots \ldots$

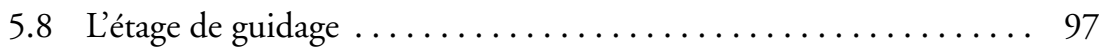


5.9 Lumière d'étalonnage $\ldots \ldots \ldots \ldots \ldots \ldots \ldots \ldots \ldots \ldots \ldots \ldots$

5.10 Les spectroscopes échelle ........................ 101

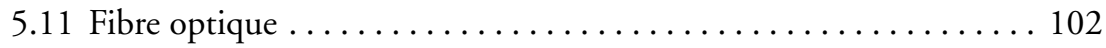

Chapitre 6 • Paramètres principaux d'un spectroscope ............ 105

6.1 Résolution et pouvoir de résolution $\ldots \ldots \ldots \ldots \ldots \ldots \ldots \ldots \ldots$

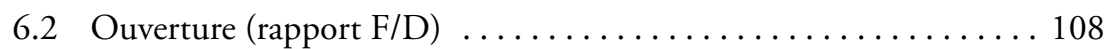

6.3 Grandissement et échantillonnage $\ldots \ldots \ldots \ldots \ldots \ldots \ldots \ldots \ldots$

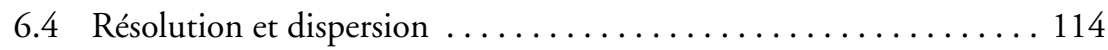

6.5 Étendue spectrale.............................. 114

6.6 Domaine spectral............................. 115

6.7 Efficacité/Rendement .......................... 115

6.8 Tirage mécanique et fixation $\ldots \ldots \ldots \ldots \ldots \ldots \ldots \ldots \ldots \ldots \ldots \ldots \ldots \ldots \ldots$

6.9 Télescope et qualité du ciel . . . . . . . . . . . . . . . . . . . . 119

6.10 Ajuster la configuration ......................... 121

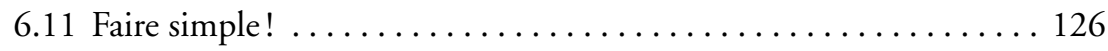

Chapitre 7 • Caméra CCD et logiciel d'acquisition............. 127

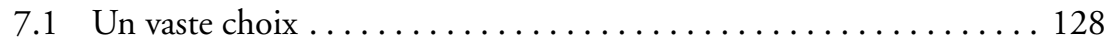

7.2 Capteur d'image ou de lumière? . . . . . . . . . . . . . 131

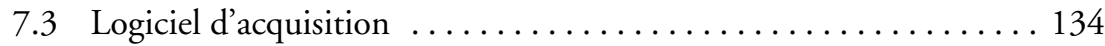

7.4 Quelques manipulations simples ................... 136

Chapitre 8 • Régler le spectroscope sur table ................. 145

8.1 Quelle source de lumière $\ldots \ldots \ldots \ldots \ldots \ldots \ldots \ldots \ldots \ldots \ldots \ldots$

8.2 Installer la caméra d'acquisition $\ldots \ldots \ldots \ldots \ldots \ldots \ldots \ldots \ldots \ldots \ldots$

8.3 Focalisation et orientation ....................... 150

8.4 Le bleu à gauche, le rouge à droite . . . . . . . . . . . . . . 152

8.5 Choisir la plage de longueur d'onde ................... 153

8.6 Régler la caméra de guidage ....................... 154

Chapitre 9 • Mesure physique et réduction de données ........... 155

9.1 Votre mission : le profil spectral . ................... 156 
9.2 Ne pas mettre la charrue avant les bœufs . . . . . . . . . . 156

9.3 Intensité et longueur d'onde ..................... 157

9.4 Distinguer Technique et Science ....................... 157

9.5 Erreur systématique et aléatoire $\ldots \ldots \ldots \ldots \ldots \ldots \ldots \ldots \ldots \ldots$

9.6 Rapport Signal/Bruit . . . . . . . . . . . . . . . . . . . . . . . . . 159

9.7 Les étapes de la réduction de données . . . . . . . . . . . . . . . 160

9.8 Catalogues d'étoiles de référence....................... 169

9.9 Une observation est un groupe d'images ............... 170

Chapitre 10 • Une première observation spectroscopique : le Soleil . . . 173

10.1 Images de référence . .......................... 174

10.2 Réduction des données ........................ 175

10.3 Étalonnage en longueur d'onde $\ldots \ldots \ldots \ldots \ldots \ldots \ldots \ldots \ldots 181$

10.4 Correction de la réponse instrumentale $\ldots \ldots \ldots \ldots \ldots \ldots \ldots 183$

Chapitre 11 • Maîtriser le télescope .......................... 189

11.1 Maitriser la monture ................................ 189

11.2 Calculer le champ de l'image ......................... 196

11.3 Repérer les mouvements du télescope ................. 198

11.4 Pointer une étoile .............................. 200

11.5 Autoguidage.................................. 204

Chapitre 12 • Installer le spectroscope sur le télescope ............... 209

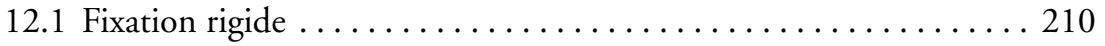

12.2 Orientation du spectroscope . . . . . . . . . . . . . . . . 210

12.3 Équilibrage et gestion des câbles ..................... 211

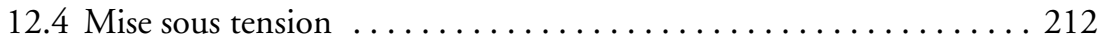

12.5 Focalisation guidage et télescope..................... 213

12.6 Dernières vérifications ............................ 215

12.7 À la nuit tombée............................... 216

Chapitre 13 • Observation spectroscopique d'une (autre) étoile . . . . . 223

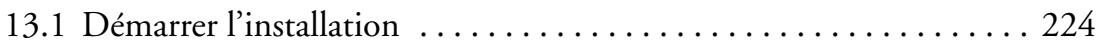


13.2 Pointez l'étoile de référence ...................... 224

13.3 Pointez l'étoile cible ............................ 224

13.4 Faites toutes les images de référence ................. 225

13.5 Réduire les données . . . . . . . . . . . . . . . . . . . . 225

13.6 Aller plus loin ............................. 227

Chapitre 14 • Mesurer la qualité du spectre ................. 231

14.1 Lire le compte rendu de calcul . ................... 232

14.2 Comparer avec les autres observateurs ................ 232

14.3 Vérifier l'étalonnage en longueur d'onde ................. 233

14.4 Disparité dans les observations . .................... 235

14.5 Mesure du rapport S/B .......................... 235

14.6 Niveau de signal pour votre instrument ............... 235

Chapitre 15 • Paré pour l'aventure ......................... 237

15.1 Session d'observation typique . .................... 237

15.2 Améliorer les observations.............................. 239

15.3 Améliorer la qualité des données .................... 239

15.4 Améliorer la productivité $\ldots \ldots \ldots \ldots \ldots \ldots \ldots \ldots \ldots \ldots \ldots \ldots \ldots \ldots$

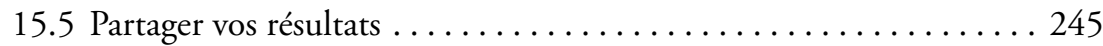

15.6 Des spectres de qualité professionnelle ................. 245

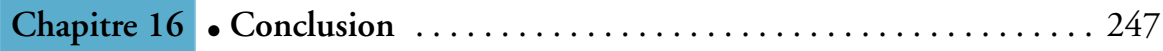

Glossaire ....................................... 249

Références..................................... 251

Note : les termes soulignés de cette manière dans le texte sont défnis dans le glossaire. 



\section{Remerciements}

Ce livre est né des nombreux échanges que j'ai pu avoir depuis plus d'une vingtaine d'années, avec la communauté des astronomes. Une communauté internationale, riche, variée, curieuse, entreprenante - passionnante.

Dans cette communauté, certains m'ont appris (beaucoup), d'autres m'ont fait confiance. Il y a des amateurs et des professionnels... beaucoup sont les deux à la fois.

J'ai une pensée particulière pour :

- Christian Buil, qui m’a tant appris. Merci pour tous ces échanges passionnés!

- Valérie Desnoux, sa compagne. Merci pour ta belle présence, et pour tous tes engagements dans Visual Spec, dans BeSS, dans les réflexions stratégiques.

- Coralie Neiner, astrophysicienne à l'Observatoire de Paris, qui nous a fait découvrir les étoiles Be, et qui est devenue une amie. Merci pour les longues discussions pour refaire le monde.

- Olivier Thizy, avec qui nous menons depuis 2006 l'aventure de l'entreprise Shelyak Instruments.

Et puis... il y a toutes ces rencontres, inépuisables sources d'énergie sur mon " chemin d'astronomie "... Maurice Abad, Agnès Acker, Jacques Adda, Evelyne Alecian, Luc Arnold, Mathieu Barthélémy, Paolo Berardi, Laurent Bernasconi, Lionel Birée, Katherine Blundell, Michel Boer, Michel Bonnement, Franck Boubault, Sylvain Bouley, Hubert Boussier, Christophe Boussin, Jacques Boussuge, Vincent 
Bouttard, Nathalie Bressand, Jean-Jacques Broussat, Yolande Buchet, Rémi Cabanac, Martine Castets, Claude Catala, Cyril Cavadore, Pascal Chambraud, Stéphane Charbonnel, Rémy Chirié, François Colas, Sophie Combe, Pierre Cruzalèbes, Jean-Luc Dauvergne, Raymond David, Steve Dearden, Bertrand De Batz, Robert Delmas, Joël Desbordes, Pierre Dubreuil, Martin Dubs, Dominique Ducerf, Nicolas Durand, Jim Edlin, Pierre Farissier, Stéphane Fauvaud, André Favaro, Christian Feghali, Paul Felenbok, Michèle Floquet, Patrick Fosanelli, Anne-Marie Galliano, Olivier Garde, Thierry Garrel, Christophe Gillier, Jean-Paul Godard, Thierry Godard, Keith Graham, Joan Guarro, Patrick Guibert, Anton Heidemann, Huib Heinrichs, Christian Hennes, Anne-Marie Hubert, Jak de Jesus, Hugo Kalbermatten, Alain Klotz, François Kugel, Olivier Labreuvoir, Robin Leadbeater, Pascal Le Du, Steve Lee, Jean-Christophe Le Floch, Thierry Lemoult, Auguste Le Van Suu, Arnaud Leroy, Bernard Leroy, Jean Lilensten, Alain Lopez, Pierre Maquart, Vincent Marik, Gérard Martineau, Jean-Pierre Masviel, Stéphane Mathis, Benjamin Mauclaire, Philippe Michel, Jacques Michelet, Richard Monnerot, Romain Montaigut, Claire Moutou, Patrick Pelletier, Sandrine Perruchot, Éric Piednoël, Jean-François Pittet, Michel Pujol, Ernst Pollmann, Franck Razafimaharo, Christian Revol, André et Sylvain Rondi, Jean-Paul Roux, Jean-Pierre Rozelot, Raymond Sadin, Èric Sarazin, Jean-Pierre Sarreyan, Carl Sawicki, Mathieu Senegas, Joël Setton, Steve Shore, Alain Soutter, Jean-Noël Terry, François-Mathieu Teyssier, Bernard Trégon, Franck Valbousquet, Céline et Sébastien Vauclair, Sylvie Vauclair, Adrien Viciana, Brigitte Zanda...

Une pensée pour mes enfants, Julien, Marion, Armand - votre fraîcheur me fait tant de bien - ainsi que pour Nathalie, avec qui on invente chaque jour notre chemin. Une pensée, enfin, pour mes deux sœurs, Marie et Cécile, ainsi que...

À papa, qui nous a donné des racines, À maman, qui nous a donné des ailes. 